\title{
Correction to: Resources for Optimal Care of Emergency Surgery
}

Michael Sugrue, Ron Maier, Ernest E. Moore, Fausto Catena, Federico Coccolini, and Yoram Kluger

\section{Correction to: \\ M. Sugrue et al. (eds.), Resources for Optimal Care of Emergency Surgery, Hot Topics in Acute Care Surgery and Trauma, https://doi.org/10.1007/978-3-030-49363-9}

The original version of the book was revised: Affiliation of one of the editors, Ernest E. Moore, has been updated. 